\title{
EFFICIENCY ENHANCEMENT IN THIN-FILM SILICON SOLAR CELLS WITH A PHOTONIC LATTICE
}

\author{
Simone Zanotto ${ }^{1,2}$, Marco Liscidini ${ }^{1}$ and Lucio Claudio Andreani ${ }^{1}$ \\ ${ }^{1}$ Dipartimento di Fisica "A. Volta" and UdR CNISM, via Bassi,6 Università degli Studi di Pavia, Italy \\ ${ }^{2}$ Scuola Normale Superiore and NEST-CNR-INFM, Pisa, Italy \\ (e-mail: $\underline{\text { simone.zanotto@sns.it, marco.liscidini@unipv.it, lucio.andreani@unipv.it) }}$
}

\begin{abstract}
We present a theoretical study of amorphous and crystalline thin-film solar cells with a periodic pattern on a sub-micron scale realized in the silicon layer and filled with silicon dioxide right below a properly designed antireflection coating. The study and optimization of the PV structure as a function of all the photonic crystals parameters allows to identify the different roles of the periodic pattern and of the etching depth in determining an increase of the absorption. From one side, the photonic crystal acts as an impendence matching layer, thus minimizing reflection of incident light over a particularly wide range of frequencies. Moreover a strong absorption enhancement is observed when the incident light is coupled into the quasi guided modes of the photonic slab. We compare the efficiency of this structure to that of PV cells characterized by the sole antireflection coating. We found a substantial increase of the short-circuit current when the parameters are properly optimized, demonstrating the advantage of a wavelength-scale, photonic-crystal based approach.
\end{abstract}

Keywords: thin film solar cell, c-Si, a-Si

\section{INTRODUCTION}

Photovoltaic conversion of solar radiant energy in electrical energy is a hot topic of the last decades, and for many years mono- or polycrystalline silicon solar cells have been the most popular devices. Usually the thickness of the absorbing layer is over 100 microns, and the cost of the silicon is more than a half of the total cost of the device; a significant reduction of fabrication costs can be achieved by reducing the absorber thickness or changing the material [1]. A variety of thin-films materials have been explored, ranging from CIGS (copper-indium-gallium-selenide) and cadmium based compounds, to semiconducting polymers and silicon itself. An advantage of silicon is the compatibility with the present chip technology, so it is plausible to develop an application of thin-film cells for direct chip supply.

In silicon thin-films the material can be microcrystalline or amorphous, where typical thickness ranges are $500-$ $1000 \mathrm{~nm}$ and $100-300 \mathrm{~nm}$, respectively [2]. These values, limited by the carrier diffusion length, are comparable with the absorption length of the photons whose energy lies between the fundamental gap and about $2.5 \mathrm{eV}$. Since the contribution of the photon flux spectrum is large in this range (considering the Sun as a blackbody of $\mathrm{T}=5800 \mathrm{~K}$ the maximum is at $0.8 \mathrm{eV}$ ), it is very important to develop light-trapping systems that can enhance the photon dwelling time in the absorbing medium. While traditional "thick" cells employ micrometer-scale patterns that base their action on geometrical optics, the limited thickness of thin-film cells requires the development of nanometer-scale, photonic based trapping structures [3-6]. These structures can couple the incoming light into the absorbing material with different mechanisms and coupling regimes, that we have investigated by varying the structure parameters [7].

\section{STRUCTURE UNDER INVESTIGATION}

The structure we considered is shown in Fig. 1. The silicon absorbing layer, of thickness $d$, lies on a silver back reflector. The front surface of silicon is patterned with a one dimensional periodic pattern with period $a$, fill factor $f=b / a$, and depth $h$. A filling of silica is assumed to reduce surface recombination losses. The top of the structure is coated with an antireflection (AR) layer, with thickness $l$ and refractive index $n$. Since our aim is to explore the role of the photonic components on the absorption, we devoted our attention to the functions of the pattern and the coating.

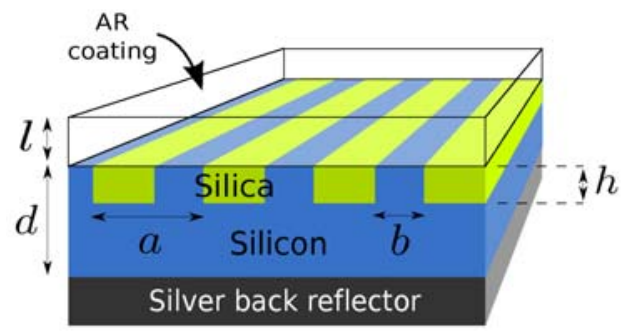

Figure 1: Structure layout.

\section{RESULTS AT NORMAL INCIDENCE}

The efficiency of the cell is estimated by means of the short circuit current, which, in the approximation that all the electron-hole pairs generated are collected by the electrodes, is simply the integral of the photon flux $\mathrm{dN} / \mathrm{dE}$ spectra weighted with the absorbance $\mathrm{A}(\mathrm{E})$ of the device:

$$
j_{\mathrm{sc}}=e \int_{0}^{\infty} A(E) \frac{d N}{d E} d E .
$$

The photonic behaviour of the structure is studied in a frame of a rigorous coupled-wave theory, with an implementation of the scattering matrix method [8,9]; once it is possible to access the Poynting vector's components, the energy absorbed in the relevant part of the structure is easily calculated from the scattering matrix itself, without the need of time-consuming realspace integrations of the fields. All the results presented in this paper are averaged over light polarizations. 
Here we describe the analysis of a microcrystalline silicon cell with thickness $d=500 \mathrm{~nm}$. In the case of a bare slab without pattern and without AR coating, but with the sole back reflector, the short-circuit current is only $8.0 \mathrm{~mA} / \mathrm{cm}^{2}$ (all the calculations refer to the blackbody spectrum in the window $[1.12,3.5] \mathrm{eV})$. On the other hand, if we consider an unpatterned silicon slab with an optimized AR coating $n=1.9, l=70 \mathrm{~nm}$ [3]), we can reach $j_{\mathrm{sc}}=10.7 \mathrm{~mA} / \mathrm{cm}^{2}$. This is the value that we take as our reference to evaluate the efficiency enhancement given by the introduction of a photonic pattern.

The first analysis consists in varying simultaneously the etching depth and the fill factor of the cell without the AR coating. The result is shown in Fig. 2 for a period $a=$ $450 \mathrm{~nm}$. The calculations performed for $a=300$ and 600 $\mathrm{nm}$ show qualitatively similar results, although the maximum $j_{\mathrm{sc}}$ is slightly smaller. Indeed, when the period is small, say $a=300 \mathrm{~nm}$, there is no diffraction light lost in the air, but also the silicon supports few diffracted waves. On the contrary, in the case of a large period ( $a=$ $600 \mathrm{~nm}$ ) there are many diffraction orders in the silicon, but part of the incident light is diffracted back to the air. For this reason in this work we fix $a=450 \mathrm{~nm}$.

In Fig. 2 it can be seen that there are two maxima, corresponding to a shallow etching $(h=80 \mathrm{~nm})$ and a deep etching $(h=300 \mathrm{~nm})$ configuration; the origin of the two maxima is an improvement of the coupling efficiency of the incident light. In both cases the shortcircuit current is about $13 \mathrm{~mA} / \mathrm{cm}^{2}$, showing an increase of about $21.5 \%$ with respect to the reference system. In the first case, a simplified picture interprets the pattern as a grating that diffracts the vertical incident rays into oblique ones, thus increasing the light path. A better picture recognizes the increased absorption as a consequence of the coupling of the radiative continuum with the slab waveguide modes, due to the periodic pattern. In the second case it is no longer possible to view the structure as a planar slab with a periodic perturbation, as it becomes rather a photonic crystal slab. The absorption is now enhanced at the energies corresponding to the photonic modes that are excited by the incoming radiation.

A further increase in the short-circuit current can be achieved by adding an AR coating directly on the patterned layer. While a rigorous optimization procedure would require an exploration of the whole parameter space $(h, f, n, l)$, it is possible to achieve good results also by optimizing one or two parameters at a time. In our case we set the etching depth at the optimal value for the shallow etching configuration $(h=80 \mathrm{~nm})$, then look for a maximum in the plane $(f, n)$, and eventually optimize $l$. The simultaneous optimization of $f$ and $n$ allows to take into account the interplay of the grating and the coating, as shown in Fig. 3. Apart from the configurations yet encountered (upper left, bare slab; upper right, slab + AR coating; left center, only pattern), in the center of the parameter space there is a configuration that gives an increase in $j_{\mathrm{sc}}$ of $25.5 \%$ with respect to the reference structure without patterning but with AR coating.

The analysis of the contour plot reveals that a separate optimization of $f$ and $n$ would not allow to find the absolute maximum. Indeed, if $f$ is first fixed to the optimal value for an uncoated structure (35\%), the sole variation of $n$ does not allow to explore the region of the maximum, as the parameters $f$ and $n$ are correlated. This correlation can be interpreted by considering the siliconsilica pattern as an effective medium, whose refractive index is an average of the indices of the two materials. When $f$ is large, the pattern has a refractive index close to that of silicon, and a good impedance match requires an AR coating with a high index; a small value of $f$ gives rise to the opposite situation. A variation of the parameter $l$ reveals that there is again the same correlation between $f$ and $n$, the maximum lies in the same position with a slight variation in the maximum achievable short-circuit current; $l=70 \mathrm{~nm}$ is the optimal value.

A similar optimization can be carried on when fixing $h$ at the deep etching value $(300 \mathrm{~nm})$, leading to an increase of $36.5 \%$; it is worth noting that this value, higher than that reported for the shallow etching, does not take into account the higher surface losses that can occur when the etching depth is increased. For this reason the shallowetching structure is probably the best compromise between maximum increase of light harvesting and minimum degradation of electronic transport properties. Similar calculations performed for a c-Si layer of 1000 $\mathrm{nm}$ thickness yield $\mathrm{j}_{\mathrm{sc}}=14.6 \mathrm{~mA} / \mathrm{cm}^{2}$ for the unpatterned + AR structure and $18.6 \mathrm{~mA} / \mathrm{cm}^{2}$ for the optimized patterned structure, with a relative increase of $27 \%$. We have also performed a similar analysis for solar cells with an amorphous silicon layer of either $100 \mathrm{~nm}$ or $300 \mathrm{~nm}$ thickness. The short-circuit current increases from 19.3 to $21.7 \mathrm{~mA} / \mathrm{cm}^{2}$ in the first case $(12.4 \%$ relative increase) and from 22.4 to $25 \mathrm{~mA} / \mathrm{cm}^{2}$ in the second case $(11.3$ relative increase). The physical properties and interpretation are similar to the case of $\mathrm{c}-\mathrm{Si}$.

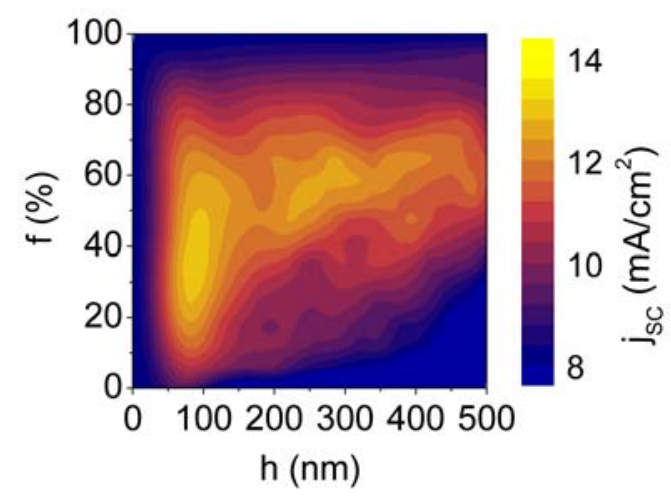

Figure 2: Short-circuit current dependence on etching depth and fill factor

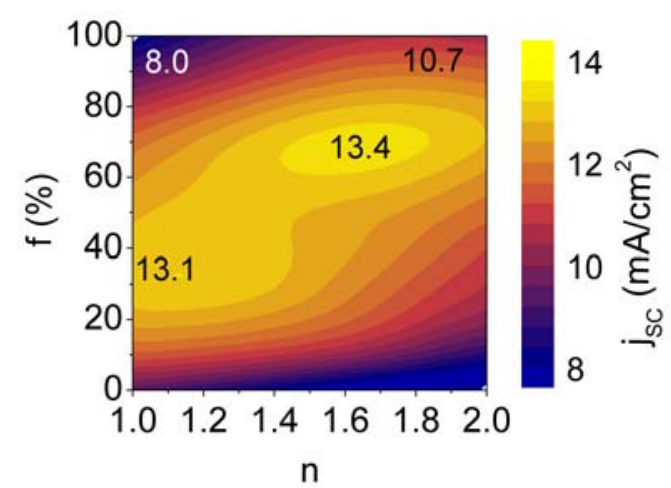

Figure 3: Short-circuit current dependence on fill factor and AR coating refractive index $(\mathrm{h}=80 \mathrm{~nm}, \mathrm{l}=70 \mathrm{~nm})$ 


\section{EFFECTS OF THE ANGLE OF INCIDENCE}

It is important to analyze the dependence of the shortcircuit current on the angle of incidence and on the orientation of the incident beam: indeed, any strong dependence on these two parameters could be detrimental for the cell performance. In Fig. 4 we show the calculated short-circuit current as a function of the incidence angle $\theta$, for the optimized c-Si structure of Fig.3. We consider two possible orientations of the incident beam: perpendicular to the grating $(\phi=0)$ and parallel to the grating $(\phi=90)$, as shown in the inset. Also, we compare the results for the grating structure with that of the unpatterned structure with AR coating. For all these cases, the decrease with the angle of incidence is due to the standard geometrical $(\cos \theta)$ factor. Surprisingly, the short-circuit current for the patterned structure is found to be weakly dependent on the orientation angle $\phi$, and the enhancement with the respect to the unpatterned structure is basically independent of the incident angle $\theta$. In other words, the efficiency enhancement of the considered grating structure is rather robust with respect to variations of the orientation and of the incidence angle. This is an interesting result, as implies that the 1D grating structure would yield the same efficiency enhancement for direct and diffuse light, without the need of any solar tracking. A fortiori, similar results are expected for 2D photonic structures, which are obviously even less anisotropic.

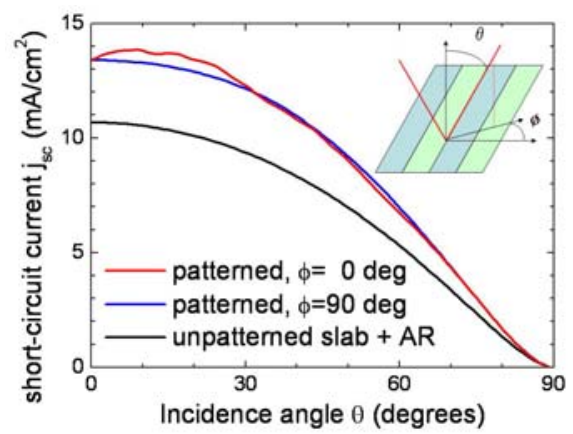

Figure 4: Short-circuit current dependence on angle of incidence, for different orientations of the incident light with respect to the grating, as shown in the inset.

\section{CONCLUSION}

We have calculated the short-circuit current for c-Si and a-Si solar cells in the presence of a photonic pattern in the silicon layer and of a properly optimized antireflection coating. The dependence of the short-circuit current on the structure parameter reveals the presence of two coupling regimes, due to the different nature of the photonic modes of shallow and deeply patterned structures. The study of an antireflection coating reveals that the pattern behaves also like an effective medium, and allows to achieve an increase in the short circuit current of up to $36.5 \%(12.4 \%)$ in the c-Si (a-Si) case with respect to the cell with AR coating and no patterning.

\section{ACKNOWLEDGMENTS}

This work was supported by CNISM through the INNESCO initiative and by Fondazione Cariplo through project 2007-5259.

\section{REFERENCES}

[1] J. Nelson, The physics of solar cells (Imperial College Press, London 2003)

[2] J. Poortmans, V. Arkhipov (editors), Thin film solar cells (Wiley, Chichester 2006)

[3] P. Bermel, C. Luo, L. Zeng, L. C. Kimerling, and J. D. Joannopoulos, Optics Express 15, (2007) 16986.

[4] M. Kroll, S. Fahr, C. Helgert, C. Rockstuhl, F. Lederer, and T. Pertsch, Phys. Stat. Sol. A 205, (2008) 2777.

[5] I. Prieto, B. Galiana, P.A. Postigo, C. Algora, L.J. Martinez, and I. Rey-Stolle, Appl. Phys. Lett. 94, (2009) 191102.

[6] Y. Park, E. Drouard, O. El Daif, X. Letartre, P. Viktorovitch, A. Fave, A. Kamnski, M. Lemiti, and C. Seassal, Optics Express 17, (2009) 14312.

[7] S. Zanotto, M. Liscidini, and L.C. Andreani, Energy Express 18, (2010) 4260.

[8] D. M. Whittaker and I. S. Culshaw, Phys. Rev. B 60, 2610 (1999).

[9] M. Liscidini, D. Gerace, L.C. Andreani, and J.E. Sipe, Phys. Rev. B 77, 035324 (2008). 